\title{
Purple to Yellow Silicone Elastomers: Design of a Versatile Sensor for Screening Antioxidant Activity
}

\author{
Ogliani, Elisa; Skov, Anne Ladegaard; A. Brook, Michael
}

Published in:

Advanced Materials Technologies

Link to article, DOI:

10.1002/admt.201900569

Publication date:

2019

Document Version

Peer reviewed version

Link back to DTU Orbit

Citation (APA):

Ogliani, E., Skov, A. L., \& A. Brook, M. (2019). Purple to Yellow Silicone Elastomers: Design of a Versatile Sensor for Screening Antioxidant Activity. Advanced Materials Technologies, 4(11), [1900569]. https://doi.org/10.1002/admt.201900569

\section{General rights}

Copyright and moral rights for the publications made accessible in the public portal are retained by the authors and/or other copyright owners and it is a condition of accessing publications that users recognise and abide by the legal requirements associated with these rights.

- Users may download and print one copy of any publication from the public portal for the purpose of private study or research.

- You may not further distribute the material or use it for any profit-making activity or commercial gain

- You may freely distribute the URL identifying the publication in the public portal 


\title{
Purple to yellow silicone elastomers: design of a versatile sensor for screening antioxidant activity
}

\author{
Elisa Ogliani, Anne Ladegaard Skov* and Michael A. Brook*
}

Elisa Ogliani, Prof. Anne Ladegaard Skov

Technical University of Denmark, Department of Chemical Engineering, 2800 Kgs. Lyngby, Denmark

E-mail: al@kt.dtu.dk

Prof. Michael A. Brook

McMaster University, Department of Chemistry and Chemical Biology, 1280 Main St. W., Hamilton, Ontario, Canada L8S 4M1

E-mail: mabrook@mcmaster.ca

Keywords: DPPH radicals, antioxidants, silicone elastomers, colorimetric sensors

Antioxidants play a key role in counteracting the adverse effects of oxidative stress in human organisms. Thus, there is a huge demand for the development of smart and convenient assays for the evaluation of antioxidant activity of synthetic and natural compounds, food samples, plant extracts, etc. Here, the design of a solid-state, flexible, and portable format of the traditional in vitro 2,2-diphenyl-1-picrylhydrazyl (DPPH) radical test is reported. For the first time, purple DPPH radicals are physically immobilized into a silicone matrix by means of a facile and rapid process. The working principle of the resulting sensor is based on the colorimetric process that is associated with the redox reaction of DPPH radicals with antioxidants. When an antioxidant reacts with the sensor, a color change from purple to yellow can be perceived by the naked-eye. The response of the sensor is investigated qualitatively and quantitatively towards food samples and selected antioxidants of different natures, and solubilized in different media. It is demonstrated that the DPPH silicone sensor is highly versatile and can be used as a ready-to-use sensor for direct colorimetric detection of antioxidants.

\section{Introduction}

Antioxidants are considered essential compounds responsible for the surveillance of human health. A delicate balance regulates prooxidants, such as reactive oxygen species (ROS), and 
antioxidant defenses in the human organism: a disruption in this balance results in oxidative stress. ${ }^{[1]}$ An excess of free radicals impairs different kind of biomolecules: lipids, proteins, and nucleic acids are all damaged by oxidation. Oxidative stress plays a critical role both in the pathogenesis of many chronic diseases, ${ }^{[2-4]}$ as well as in the aging process. ${ }^{[5]}$ Antioxidants are in charge of scavenging and deactivating free radicals before they can elicit adverse effects via oxidative damage. The human organism has an endogenous antioxidant defense system, which is supported by the additional intake of exogenous antioxidants deriving from dietary supplements. ${ }^{[6]}$ The importance of antioxidants in fighting the pathological conditions related to oxidative stress and their resulting beneficial effects on health are well-established. Therefore, a lot of attention is focused on which compounds have the highest antioxidant activity or which foods contain the highest amount of antioxidants.

Many in vitro analytical assays have been developed to measure the antioxidant activity of compounds and food/beverage extracts, and they are based on different mechanisms. For instance, the oxygen radical absorbance capacity (ORAC) ${ }^{[7]}$ and total peroxyl radical trapping antioxidant parameter (TRAP) ${ }^{[8]}$ are analytical assays based on a hydrogen atom transfer (HAT) mechanism. The 2,2-diphenyl-1-picrylhydrazyl (DPPH) ${ }^{[9]}$ and the azinobis (3ethylbenzothiazoline-6-sulfonic acid (ABTS) ${ }^{[10]}$ assays are based on an electron transfer (ET) mechanism, or on a mixed-mode (HAT/ET) mechanism. ${ }^{[11]}$ Of the available reagents, the DPPH radical benefits from remarkable stability due to steric hindrance and the push-pull effect exerted by the functional groups on the divalent $\mathrm{N}$ atom; ${ }^{[12]}$ it has an intense purple color in solution (maximum absorption band at a wavelength of $\sim 515 \mathrm{~nm}$ ). When a hydrogen donor, such as an antioxidant, reduces DPPH a change in color from purple to pale yellow is observed due to the formation of its hydrazinic form (Figure 1); the decrease in the absorption band at $515 \mathrm{~nm}$ can be easily monitored through a spectrophotometer. For these reasons, the DPPH assay is extensively used in laboratories to measure the antioxidant ability of compounds. ${ }^{[13]}$ 
Solid-state colorimetric sensors provide a very competitive alternative to conventional solutionbased analytical assays, as they offer the great advantages of being facile, rapid, portable and cost-effective. ${ }^{[14]}$ The solid-state sensing technology allows for the naked-eye and in-situ detection of the results, and it does not require large volume of solvents for the test. DPPH assays are progressively moving towards the new direction of solid-state colorimetric testing. In the literature, for instance, a paper-based DPPH assay has been validated by Sirivibulkovit et al., ${ }^{[15]}$ and a PVC film with immobilized DPPH radicals has been developed by Steinberg et

$a l .{ }^{[16]}$ However, the mentioned approaches present some limitations: the DPPH reagent is not immobilized but only deposited on a solid surface, ${ }^{[15,17]}$ or the fabrication process of the sensing platform is time consuming. ${ }^{[16,18]}$

When designing a colorimetric sensor, the choice of a suitable host matrix for the indicator molecules is crucial. Silicone elastomers have been widely used as matrices for entrapping indicator dyes to produce optical chemosensors, e.g., for gas sensing. ${ }^{[19]}$ They offer many advantages such as versatility, easy processability, optical transparency, and flexibility.

In this study, the use of DPPH radicals that are physically immobilized as a uniform dispersion into a silicone matrix is reported. The silicone colorimetric sensor presented here can be fabricated in a rapid and facile manner from commercial materials, and the resulting elastomer can be used as a solid-state and ready-to-use sensor for direct colorimetric detection of antioxidants. The response of the sensor is investigated qualitatively and quantitatively towards selected antioxidants of different natures and solubilized in different media.

\section{Results}

\subsection{DPPH silicone elastomers as colorimetric sensors}

DPPH was entrained in condensation cured silicone elastomers (Figure 2a) using a very easy preparative process. The maximum concentration of DPPH radicals was found to be $0.03 \mathrm{wt} \%$. Above this value, DPPH precipitates/aggregates scattered light, as could be seen either with the 
naked eye or by use of optical microscopy. The uncured silicone matrix with homogeneously dispersed DPPH radicals was cast as a freestanding elastomer film (Figure $\mathbf{2 b}, \mathbf{c}$ ) and the samples were cured overnight at room temperature. When curing was complete, cylindrical DPPH silicone elastomers were cut with a hole puncher and arranged to form a screening platform (Figure 3) which is ready-to-use for the direct detection of antioxidant compounds of interest. Initially, three different antioxidants (Figure S1) were used as a reference to investigate the response of the sensor: ascorbic acid (vitamin C), $\alpha$-tocopherol (vitamin E), and butylated hydroxytoluene (BHT), which are commonly used as reference standards for the assessment of antioxidant properties of compounds. ${ }^{[20]}$ Drops of antioxidant solutions at different concentrations were placed on the surface of the DPPH silicone colorimetric sensor and the discoloration caused by the reduction of DPPH radicals was monitored over time. BHT and vitamin $\mathrm{E}$ in isopropanol caused a fast change in the color of the sensor from purple to yellow, leading to an extent of discoloration that correlated with the concentration of the solutions (Figure 3). In response to aqueous vitamin $\mathrm{C}$ solutions, the sensor started to develop a pale yellow color only $\sim 5$ minutes after contact with the antioxidant solution. Even though the sensor responded positively to vitamin $\mathrm{C}$ in solution, the discoloration induced by vitamin C did not show a correlation with the antioxidant concentration in solution, in contrast with the case of vitamin $\mathrm{E}$ and BHT.

\subsection{Microwell plates coated with DPPH silicone elastomers}

DPPH silicone elastomer can be easily coated on the bottom of the wells of a microwell plate. In this way, the DPPH silicone coated microplate is ready-to-use for the quantitative assessment of the DPPH radical scavenging activity of the desired compounds through spectrophotometric techniques (Figure 4), including in standard high throughput systems.

Response curves of the sensor to the antioxidants were made by both varying the concentration of DPPH entrained in the silicone $(0.05,0.03$, and $0.01 \mathrm{wt} \%)$, and the concentration of the antioxidants pipetted into the wells for the analysis. The antioxidants selected for the test were 
vitamin $\mathrm{E}$, vitamin $\mathrm{C}$, BHT, and quercetin, which is a flavonoid that is known to quench DPPH radicals. ${ }^{[12]}$ DPPH radicals exhibit a characteristic absorption band at $514 \mathrm{~nm}$. Changes in the signal from DPPH entrained in the silicone sensor at $514 \mathrm{~nm}$ was measured over time (180 min) by means of a UV-Vis spectrophotometer, and the response curves of the antioxidants are shown in Figure 5a (the response curves of the sensors filled with 0.01 and 0.05 wt\% DPPH radicals are shown in Figure $\mathbf{S 5})$.

The limit of detection of the sensor for solutions of vitamin E, vitamin C, and BHT and the reproducibility of the test expressed as relative standard deviation (\%RSD) are reported in Table S1 and range from 60-120 $\mu \mathrm{M}$. The radical quenching activity of the antioxidants was expressed as DPPH scavenging effect (\%) and DPPH remaining (\%) (calculated values of \%DPPH scavenging effect and \%DPPH remaining are shown in Figure S6 and S7 in the Supporting Information, respectively). In addition, a 24-microwell plate coated with DPPH silicone was used to determine whether the thickness of the elastomer sensor influences its response to different antioxidants. A silicone formulation with $0.03 \mathrm{wt} \%$ entrained DPPH radicals was coated on the bottom of the wells with five different thicknesses and the response to a $1 \mathrm{mM}$ solution of vitamin $\mathrm{C}$, BHT, and vitamin $\mathrm{E}$ was measured over time (180 min). As shown in Figure S8, the value of \%DPPH scavenging effect decreases with increasing thickness of the elastomer for all the tested antioxidants. With increasing thickness of the elastomer, both the extraction of DPPH radicals entrained in the sensor by the solvent and the migration of the antioxidants into the body of the sensor are less efficient. In other words, diffusion through the silicone body plays a major role in the rate at which the assay can be performed. On the other hand, when testing a sensor with thickness of $200 \mu \mathrm{m}$ and below, the absorbance at $514 \mathrm{~nm}$ measured by UV-Vis spectrophotometer is too low to obtain a consistent value of the \%DPPH scavenging effect. Based on these findings, the most convenient and practical thicknesses in this profile were found to be between 400 and $500 \mu \mathrm{m}$.

\subsection{Real food and beverage samples}


The DPPH silicone sensor was also used to evaluate the antioxidant activity of unmodified food or beverage samples that are known to possess antioxidant properties. ${ }^{[21-24]}$ Response curves of the sensor to the samples (Figure 6b) were measured with a spectrophotometer by using a 24microwell plate coated with DPPH silicone, as discussed in the previous paragraph. Figure 6a shows the discoloration of DPPH silicone sensor after contact with sunflower oil, olive oil, black tea, green tea, black coffee, and white wine, respectively.

\subsection{Stability studies}

DPPH radicals in solution are known to suffer from decomposition issues ${ }^{[25]}$ and, therefore, the degree of long-time storage could be expected to be limited: when DPPH radicals in solutions are exposed to light, oxygen and $\mathrm{pH}$, a decrease in absorbance is observed over time. In order to investigate the stability of the DPPH silicone sensor under normal storage conditions, the absorbance of the sensor and its response towards antioxidants were measured over time using two different storage conditions: room temperature and refrigerator temperature (DPPH dry reagent and DPPH solutions are commonly stored at $\left.+4-6^{\circ} \mathrm{C}\right)$. During the stability studies, the microwell plates were wrapped in aluminum foil and stored in the dark to avoid light-induced degradation of the radicals, but were not protected from air. As expected, the stability was improved significantly when the sensor is kept in the refrigerator (Table S2). In both storage conditions, no significant decrease in absorbance was detected after one day of storage. After one week storage of the sensor at room temperature a decrease in absorbance of $8 \%$ and a decrease in response of $16.4 \%$ were observed; when stored at refrigerator temperatures a decreases in absorbance of $3.5 \%$ and a decrease in response of $8.5 \%$ were observed.

\section{Discussion}

It is shown that the silicone colorimetric sensor with immobilized DPPH radicals presented here can be used for a facile and rapid naked-eye analysis of antioxidants, either for a positive/negative screening, or to discriminate different concentrations of antioxidant. Since only few microliters of solution $(20-40 \mu \mathrm{L})$ are required to perform the assay, the solid-state 
DPPH sensing platform allows for a tremendous reduction in sample volumes over traditional DPPH assays. ${ }^{[20]}$ The color change that evolves after dropwise application of an antioxidant solution on the surface of the sensor can be potentially quantified by color intensity measurements. This type of approach is becoming more and more popular in diagnostics and chemical and biochemical assays, due to its simplicity and cost-effectiveness. ${ }^{[26,27]}$

The concentration of DPPH radicals embedded in the silicone and the thickness of the sensor films were adjusted in order to observe an optimal development in the color change that could be sensed visually. For this purpose, a film thickness ranging from $350 \mu \mathrm{m}$ to $500 \mu \mathrm{m}$ and a concentration of DPPH radicals in the sensor of $0.03 \mathrm{wt} \%$ were found to be optimal. Different reaction rates of the antioxidants with DPPH radicals entrained in the silicone elastomers were observed. These differences are attributed both to rates of migration of the antioxidant through the silicone and rates of reaction with DPPH. While vitamin E in isopropanol induced a discoloration of the sensor shortly after the contact, BHT in isopropanol showed a slower initial reactivity, followed by a fast discoloration developing $\sim 10$ minutes after the contact with the sensor. One of the factors that strongly affects DPPH reactivity with antioxidant compounds is the steric accessibility to the radical site. ${ }^{[28]}$ Thus, a slower reactivity shown by BHT compared to vitamin E can be partially explained by the considerable steric hindrance of BHT (Figure S1) that affects the reaction rate. ${ }^{[29]}$ These two antioxidants are at least partly soluble in silicones and readily dissolve in isopropanol; silicones readily swell with isopropanol. Thus, the noted differences in rates between the antioxidants are ascribed to relative reactivity, not solubility or mobility through the silicone.

In contrast to $\mathrm{BHT}$ and vitamin $\mathrm{E}$, vitamin $\mathrm{C}$ in water did not induce an extent of discoloration that correlates with the concentration of the solutions. This observation can be supported by two possible explanations. First of all, the hydrophilic vitamin $\mathrm{C}$ can only react at the surface of the hydrophobic silicone sensor - it will not diffuse into the body of the silicone elastomer. Secondly, the aqueous solution is not capable of extracting the DPPH embedded in the sensor, 
due to the poor solubility of DPPH in water. Thus, the efficiency of this sensor for vitamin C is compromised by lack of solubility/swellability in water.

The case of quercetin requires a separate discussion. As reported by Foti et al.,$^{[30]}$ monitoring the reaction of DPPH with quercetin using ordinary spectrophotometers can be misleading, since the UV-vis absorption spectra of DPPH and the oxidation products of quercetin (Figure S4) are similar. Therefore, the decrease in absorbance of DPPH at $514 \mathrm{~nm}$ (that represents the DPPH radical scavenging activity of quercetin) can be misinterpreted. Nevertheless, as shown in Figure 4, the response of the sensor to quercetin can be clearly detected by the naked-eye and the color change correlates to the concentration of antioxidant in solution.

The main advantage of the DPPH silicone sensor is its versatility. First of all, the colorimetric sensor is responsive towards compounds of different nature, such as hydrophilic (e.g. vitamin $\mathrm{C}$, in water) as well as hydrophobic (e.g. vitamin E, in alcohol) compounds, albeit at different rates. Secondly, it is possible to perform the test in many different media. Alcoholic solvents are usually required to perform the traditional DPPH assay, due to the very poor solubility of DPPH in water. Many strategies have been developed to overcome this issue, such as solubilization of DPPH in water using $\beta$-cyclodextrin, ${ }^{[31]}$ or the use of water-soluble derivatives of DPPH. ${ }^{[32]}$ The DPPH colorimetric silicone sensor presented in this work can be used to evaluate the antioxidant ability of compounds in aqueous as well as organic media. With respect to the analysis of compounds solubilized in an aqueous medium, the use of a relatively thin sensor film is recommended.

The ability to measure antioxidant activity qualitatively and quantitatively without the need for pretreatment provides a real advantage to DPPH silicone sensors over traditional routes. The practicality of the DPPH colorimetric sensor is not only endorsed by its compatibility with the analysis of real food and beverage samples, but also for its stability. Even though a progressive degradation over time is detected, the DPPH silicone sensor shows a significant improvement 
in the stability in comparison to other methods for immobilizing DPPH radicals previously reported in literature. ${ }^{[15,16]}$

Silicone elastomers have been advantageously used in many applications because of the ease of fabrication, transparency, and the inherent flexibility given by the silicone elastomeric network. They may be prepared in flat sheets, as described here, but also in much more complex forms, including via 3D printing. ${ }^{[33]}$ The DPPH elastomer here constitutes an example of a responsive polymer, ${ }^{[34,35]}$ which are of increasing value in a wide variety of (bio)analytical assays, including microfluidic technologies ${ }^{[36,37]}$ that are used to measure antioxidant activity of target molecules. ${ }^{[38]}$ This practical, easy to fabricate sensor may be prepared in a variety of physical formats, and can be optimized to test for antioxidant activity in a variety of liquid media. The sensor allows for rapid qualitative assessment - including in situ testing of different food and beverage samples, ranging from samples in oil phase (e.g., olive oil), to samples in aqueous media (e.g., tea), without need of any special pre-treatment - or can be optimized for more quantitative analyses. Work to develop sensors that can discriminate between different antioxidants, by exploiting the observed differences in rates of reductions, is ongoing.

\section{Conclusion}

In this study, a new colorimetric solid-state sensor for rapid, cost-effective, and facile screening of antioxidant activity was developed. DPPH radicals were physically immobilized into a silicone matrix by means of a simple process. Once silicone cure has taken place, the sensor is ready-to-use for the in situ assessment of the presence of antioxidant compounds (yes/no test). Both the quantity of solvents needed for the test and the preparation time for the assay are reduced tremendously: given these advantages, the DPPH silicone sensor represents a competitive alternative to the traditional in vitro DPPH assay.

It was demonstrated that the remarkable versatility of the DPPH silicone sensor allows for the analysis of compounds of different nature, solubilized in both organic as well as aqueous media. Different reference compounds, such as vitamins (vitamin E, vitamin C), the synthetic 
antioxidant BHT, the flavonoid quercetin, and non-pretreated food/beverage samples were used to test the efficiency of the DPPH silicone sensor. When the redox reaction occurs between the antioxidants and the DPPH radicals entrained in the silicone, it is possible to observe a color change from purple to yellow that develops within 5 10 minutes.

The extent of the change in color of the sensor correlates with the concentration of antioxidant applied. When prepared within the wells of a microplate reader, it is possible to evaluate quantitatively the antioxidant activity of compounds using a spectrophotometer. The response of the sensor is measured by monitoring the decay in absorbance over time caused by contact with antioxidant solutions with different concentrations. Thus, DPPH silicone sensors can be employed for both qualitative and quantitative evaluation of antioxidant activity of desired compounds or food/beverage samples.

\section{Experimental Section}

Reagents: Telechelic silanol-terminated PDMS $\left(\mathrm{M}_{\mathrm{w}}=\sim 17000 \mathrm{~g} \mathrm{~mol}^{-1}\right.$, as determined by size exclusion chromatography) was purchased from Wacker Chemie AG (Germany). Methyltrimethoxysilane and dibutyltin diacetate catalyst were purchased from Sika AG (Switzerland). 2,2-Diphenyl-1-picrylhydrazyl (DPPH) radical, 2-propanol ( $\geq 99.8 \%$ ), $\alpha$ tocopherol, ascorbic acid, butylated hydroxytoluene, and quercetin were purchased from Sigma-Aldrich (US). Absolute ethanol was purchased from VWR Chemicals (US).

Food and beverage samples: Olive oil (Monini), sunflower oil (Coop), black coffee (Douwe Egberts), and white wine (Chardonnay, France) were purchased in a local supermarket (region of Copenhagen, Denmark) and used without any preliminary treatment. Homemade olive oil was supplied by a local olive oil producer from the Lazio region (Italy). Tea samples were prepared by pouring $200 \mathrm{~mL}$ of boiling water over $2 \mathrm{~g}$ of green and black tea leaves (origin: China), respectively. The tea was steeped for either $10 \mathrm{~min}$ or $24 \mathrm{~h}$ and then filtered. Preparation of the DPPH silicone formulation: Silicone elastomers were synthesised via a condensation reaction between the telechelic silanol-terminated PDMS and the 
methyltrimethoxysilane cross-linker, catalysed by tin (dibutyltin diacetate). An attempt was made to prepare DPPH-containing silicone elastomers via a platinum-catalyzed hydrosilylation reaction, but the DPPH radicals were found to be incompatible with the addition curing formulation. The stoichiometric ratio (molar ratio of methoxy groups over silanol groups, $r$ ) used to cross-link the elastomers was $r=20$. Firstly, cross-linker $\left(0.5 \mathrm{~g}, 3.510^{-3} \mathrm{~mol}\right)$ and DPPH (1.5 $\left.10^{-3} \mathrm{~g}, 3.810^{-6} \mathrm{~mol}\right)$ were mixed uniformly using a dual asymmetric centrifuge (SpeedMixer DAC 150 FVZ-Kat) at $3500 \mathrm{rpm}$ for $4 \mathrm{~min}$. Subsequently, PDMS (4.5 g, 2.66 10 $\left.{ }^{4} \mathrm{~mol}\right)$ and tin catalyst $(0.012 \mathrm{~g}, 0.25 \mathrm{wt} \%$ of the total formulation) were added to the mixture which was mixed again at $3500 \mathrm{rpm}$ for $4 \mathrm{~min}$. As a result, DPPH appeared to be uniformly distributed within the silicone formulation.

Fabrication of the colorimetric sensor: In order to produce the colorimetric sensor, the DPPH silicone formulation was cast with an automatic film applicator (Elcometer 4340) and knife (Elcometer 3580) at a fixed thickness (e.g., at $\sim 350 \mu \mathrm{m}$ ) and cured in the dark at room temperature overnight. When curing was complete, the silicone elastomer appeared as a transparent purple freestanding film with homogeneously dispersed DPPH radicals. For quantitative assessment of the radical quenching activity of the antioxidants, the DPPH silicone formulation was coated on the bottom of the wells of a 24-microwell plate. An identical amount of silicone elastomer ( $0.190 \mathrm{~g})$ was transferred into each well, ensuring the same final thickness of approximately $500 \mu \mathrm{m}$ for all the resulting sensor discs when curing was complete.

Fourier transform infrared spectroscopy: Attenuated total reflectance Fourier transform infrared (ATR FTIR) spectra in the range of $4000-350 \mathrm{~cm}^{-1}$ were recorded on a Nicolet iS50 ATR spectrometer with a diamond crystal from Thermo Scientific.

Scanning electron microscopy: Scanning electron microscopy (SEM) and microanalysis were performed with an FEI Quanta 200E-SEM environmental scanning electron microscope, equipped with a field emission gun. The surface was visualised in a low vacuum, using water vapour as auxiliary gas at a pressure of $150 \mathrm{~Pa}$. A mixture of secondary and back-scattered 
electrons, generated by the sample surface, was detected with the large field detector for an incident electron beam of spot 3 accelerated to $10 \mathrm{keV}$. The elemental composition of the samples was determined by energy dispersive $\mathrm{x}$-rays (EDX) with an Oxford Instruments 80 mm2 X-Max silicon drift detector $\mathrm{Mn} \mathrm{K} \alpha$ resolution at $124 \mathrm{eV}$, also in a low vacuum (150 Pa) with a $500 \mu \mathrm{m}$ pressure-limiting aperture x-ray cone. Microanalysis data acquisition and quantification were performed with the Oxford Instruments Aztec program version 3.1.

Preparation of the antioxidant solutions: Stock solutions $(2 \mathrm{mM})$ of test antioxidants were prepared in ethanol or isopropanol ( $\alpha$-tocopherol, butylated hydroxytoluene, and quercetin) or deionized water (ascorbic acid) and diluted to progressively lower concentrations for analysis $(1,0.5$, and $0.25 \mathrm{mM})$

Response curves of the DPPH silicone sensor: A Polar Star Omega (BMG Labtech) spectrophotometer was used to measure the response of the sensor to antioxidants over time. 24-microwell plates coated with DPPH silicone were used to perform the experiments. The absorbance spectra $(\lambda=220-1000 \mathrm{~nm})$ of the sensors were recorded before and after the measurements. For the response curves, antioxidant solution $(500 \mu \mathrm{L})$ in different concentrations were transferred into the wells coated with DPPH silicone elastomers and the absorbance at $514 \mathrm{~nm}$ was recorded every $5 \mathrm{~min}$ for $180 \mathrm{~min}$. UV-Vis absorbance is expressed as normalized optical density (O.D.).

The detection limit of the sensor for the tested antioxidants was assessed by measuring the response of the sensor (DPPH loading of $0.03 \mathrm{wt} \%$ ) to progressively lower concentrations of the antioxidant solutions (from a concentration of $2 \mathrm{mM}$ to $15 \mu \mathrm{m}$ ). The limit of detection is expressed as the lowest concentration of the antioxidant solution that induces a response in the sensor that can be detected by means of UV-Vis spectrophotometer.

The reproducibility of the test was assessed for 10 silicone sensors (DPPH loading of $0.03 \mathrm{wt} \%$ ) prepared following the same procedure by measuring the response (normalized O.D. at $514 \mathrm{~nm}$ ) 
of the sensors after 30 minutes reaction with solutions of vitamin $\mathrm{C}$, vitamin $\mathrm{E}$, and BHT (0.5 $\mathrm{mM})$. The reproducibility values are reported as relative standard deviations.

The radical quenching activity of the antioxidants was calculated using the fixed reaction time method and the steady state saturation method, ${ }^{[20]}$ expressed respectively by Equation 1 and 2:

1) $\mathrm{DPPH}$ scavenging effect $(\%)=\frac{\left(A_{0}-A_{1}\right)}{A_{0}} 100$

( $A_{0}=$ initial absorbance, $A_{l}=$ absorbance after $\left.30 \mathrm{~min}\right)$

2) $\mathrm{DPPH}$ remaining $(\%)=\frac{A_{f}}{A_{i}} 100$

( $A_{i}=$ initial absorbance, $A_{f}=$ absorbance at the steady state).

Stability studies: To perform stability studies, two 96-microwell plates were coated with DPPH silicone and stored for one month at room temperature and at refrigerator temperature $\left(+4{ }^{\circ} \mathrm{C}\right)$, respectively. The absorbance of the sensor and its response to a freshly prepared solution of vitamin $\mathrm{E}$ (concentration of $1 \mathrm{mM}$ in ethanol) were measured 7, 14, and 30 days following the preparation day, respectively. The decrease in absorbance of the sensor over time was calculated based on the initial absorbance. The decrease in response of the sensor over time was calculated based on the \%DPPH scavenging effect of $1 \mathrm{mM}$ solution of vitamin E measured the day after preparation of the sensor.

\section{Acknowledgements}

The authors acknowledge Villum Fonden, Otto Mønsted Fonden and the Natural Sciences and Engineering Research Council of Canada for funding the project.

Received: ((will be filled in by the editorial staff)) Revised: ((will be filled in by the editorial staff)) Published online: ((will be filled in by the editorial staff))

\section{References}

[1] A. M. Pisoschi, A. Pop, Eur. J. Med. Chem. 2015, 97, 55.

[2] T. Rahman, I. Hosen, M. M. Towhindul Islam, H. U. Shekhar, Adv. Biosci. Biotechnol. 
2012, 3, 1 .

[3] Y. Feng, X. Wang, Oxid. Med. Cell. Longev. 2012, 2012, 1.

[4] E. Meagher, D. J. Rader, Trends Cardiovasc. Med. 2001, 11, 162.

[5] M. F. Beal, Free Radic. Biol. Med. 2002, 32, 797.

[6] V. Sindhi, V. Gupta, K. Sharma, S. Bhatnagar, R. Kumari, N. Dhaka, J. Pharm. Res. 2013, 7, 828 .

[7] G. Cao, H. M. Alessio, R. G. Cutler, Free Radic. Biol. Med. 1993, 14, 303.

[8] D. D. M. Wayner, G. W. Burton, K. U. Ingold, S. Locke, FEBS Lett. 2002, 187, 33.

[9] S. M. Blois, Nature 1958, 181, 1199.

[10] R. Re, N. Pellegrini, A. Proteggenete, A. Pannala, M. Yang, C. Rice-Evans, Free Radic. Biol. Med. 1999, 26, 1231.

[11] R. Apak, M. Özyürek, K. Güçlü, E. Çapanoglu, J. Agric. Food Chem. 2016, 64, 1028.

[12] M. C. Foti, J. Agric. Food Chem. 2015, 63, 8765.

[13] W. Brand-Williams, M. E. Cuvelier, C. Berset, LWT - Food Sci. Technol. 1995, 28, 25.

[14] A. Piriya V.S, P. Joseph, K. Daniel S.C.G., S. Lakshmanan, T. Kinoshita, S. Muthusamy, Mater. Sci. Eng. C 2017, 78, 1231.

[15] K. Sirivibulkovit, S. Nouanthavong, Y. Sameenoi, Anal. Sci. 2018, 34, 795.

[16] I. M. Steinberg, S. Milardović, Talanta 2007, 71, 1782.

[17] M. A. Hidayat, A. Fitri, B. Kuswandi, Acta Pharm. Sin. B 2017, 7, 395.

[18] Y. H. Ahn, J. S. Yoo, S. H. Kim, Bull. Korean Chem. Soc. 2010, 31, 2557.

[19] G. Orellana, J. López - Gejo, B. Pedras, Silicone Films for Fiber - Optic Chemical Sensing; Scrivener Publishing LLC: Salem, Massachusetts, USA, 2014; Vol. 3.

[20] K. Mishra, H. Ojha, N. K. Chaudhury, Food Chem. 2012, 130, 1036.

[21] A. Foscolou, E. Critselis, D. Panagiotakos, Maturitas 2018, 118, 60.

[22] F. Fine, C. Brochet, M. Gaud, P. Carre, N. Simon, F. Ramli, F. Joffre, Eur. J. Lipid Sci. Technol. 2016, 118, 680. 
[23] L. Xing, H. Zhang, R. Qi, R. Tsao, Y. Mine, J. Agric. Food Chem. 2019, 67, 1029.

[24] R. Romanet, C. Coelho, Y. Liu, F. Bahut, J. Ballester, M. Nikolantonaki, R. D. Gougeon, Molecules 2019, 24, 1353.

[25] B. Ozcelik, J. H. LEE, D. B. MIN, J. Food Sci. 2003, 68, 487.

[26] M. Ornatska, E. Sharpe, D. Andreescu, S. Andreescu, Anal. Chem. 2011, 83, 4273.

[27] W. Wang, W. Y. Wu, X. Zhong, W. Wang, Q. Miao, J. J. Zhu, Biosens. Bioelectron. 2011, 26, 3110 .

[28] K. Pyrzynska, A. Pȩkal, Anal. Methods 2013, 5, 4288.

[29] J. Xie, K. M. Schaich, J. Agric. Food Chem. 2014, 62, 4251.

[30] M. C. Foti, C. Daquino, G. A. Dilabio, K. U. Ingold, Org. Lett. 2011, 13, 4826.

[31] I. Nakanishi, K. Ohkubo, K. Imai, M. Kamibayashi, Y. Yoshihashi, K. I. Matsumoto, K. Fukuhara, K. Terada, S. Itoh, T. Ozawa, S. Fukuzumi, Chem. Commun. 2015, 51, 8311.

[32] G. Ionita, V. E. Sahini, G. Semenescu, P. Ionita, Acta Chim. Slov. 2000, 47, 111.

[33] S. Zheng, M. Zlatin, P. R. Selvaganapathy, M. A. Brook, Addit. Manuf. 2018, 24, 86.

[34] C. Aulin, I. Varga, P. M. Claesson, L. Wågberg, T. Lindström, Langmuir 2008, 24, 2509.

[35] E. Cabane, X. Zhang, K. Langowska, C. G. Palivan, W. Meier, Biointerphases 2012, 7, 1.

[36] J. C. Mcdonald, D. C. Duffy, J. R. Anderson, D. T. Chiu, Electrophoresis 2000, 21, 27.

[37] T. Rambarran, F. Gonzaga, A. Fatona, M. Coulson, S. Saem, J. Moran-Mirabal, M. A. Brook, J. Polym. Sci. Part A Polym. Chem. 2018, 56, 589.

[38] J. Lee, J. R. Choi, S. K. Ha, I. Choi, S. H. Lee, D. Kim, N. Choi, J. H. Sung, Lab Chip 2014, 14, 2948. 


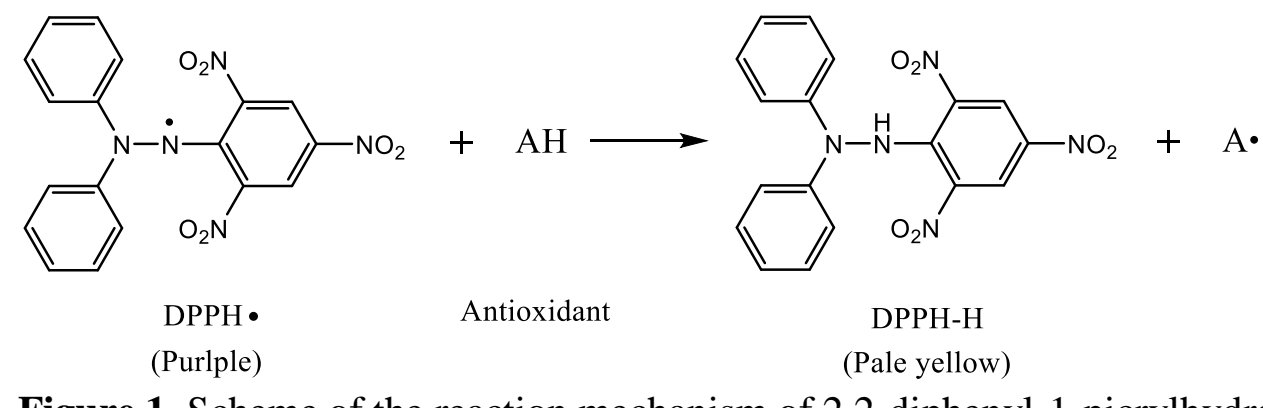

Figure 1. Scheme of the reaction mechanism of 2,2-diphenyl-1-picrylhydrazyl (DPPH) radical with antioxidants $(\mathrm{AH})$.

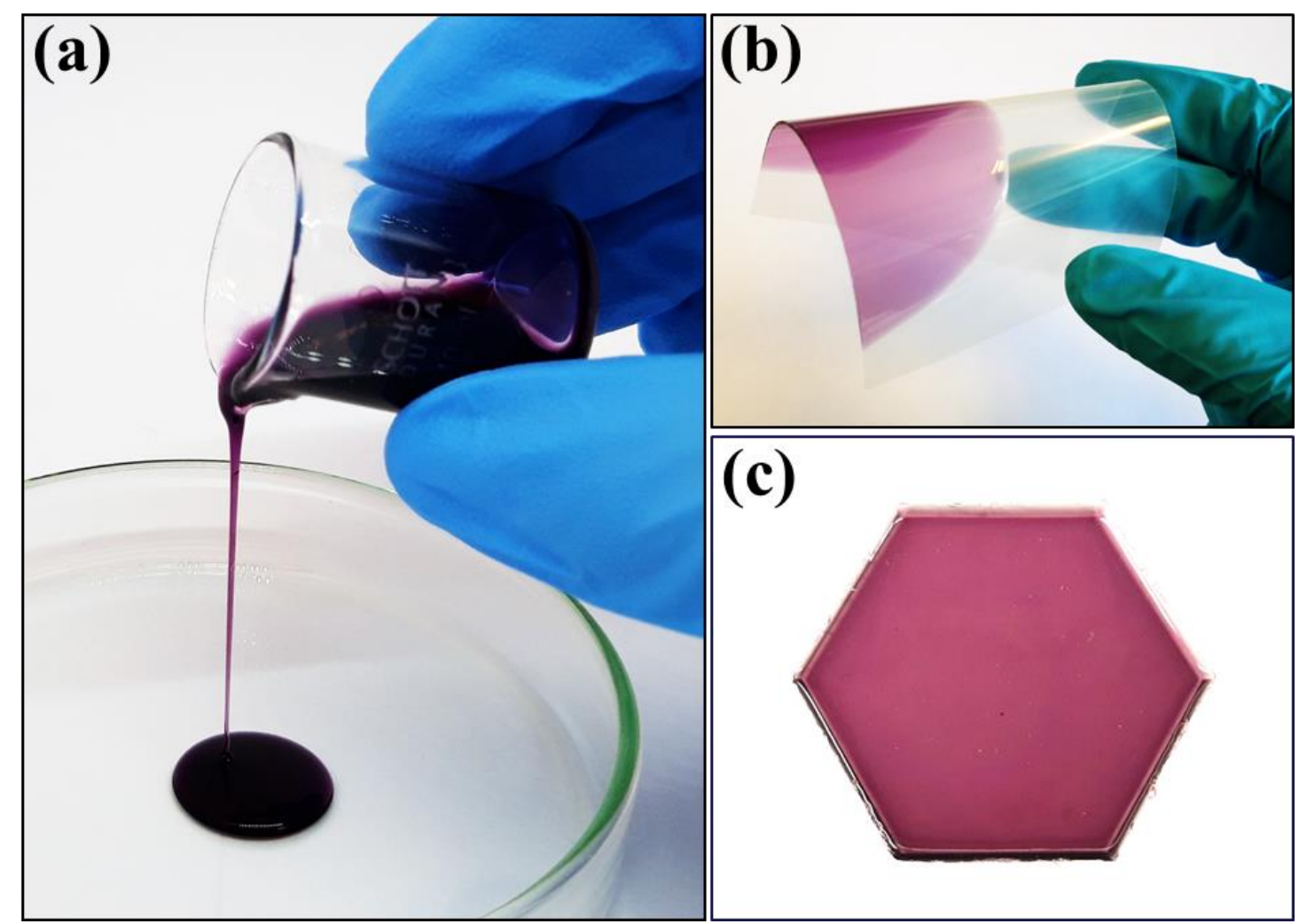

Figure 2. a) DPPH radicals can be dispersed within a silicone formulation. b) DPPH silicone formulation is cast on a polyester support (from which, optionally, it may be peeled) to form a flexible elastomeric film. c) When curing is complete, DPPH silicone is a free-standing elastomer and can be cut in the desired shape. 


\section{BEFORE THE TEST}
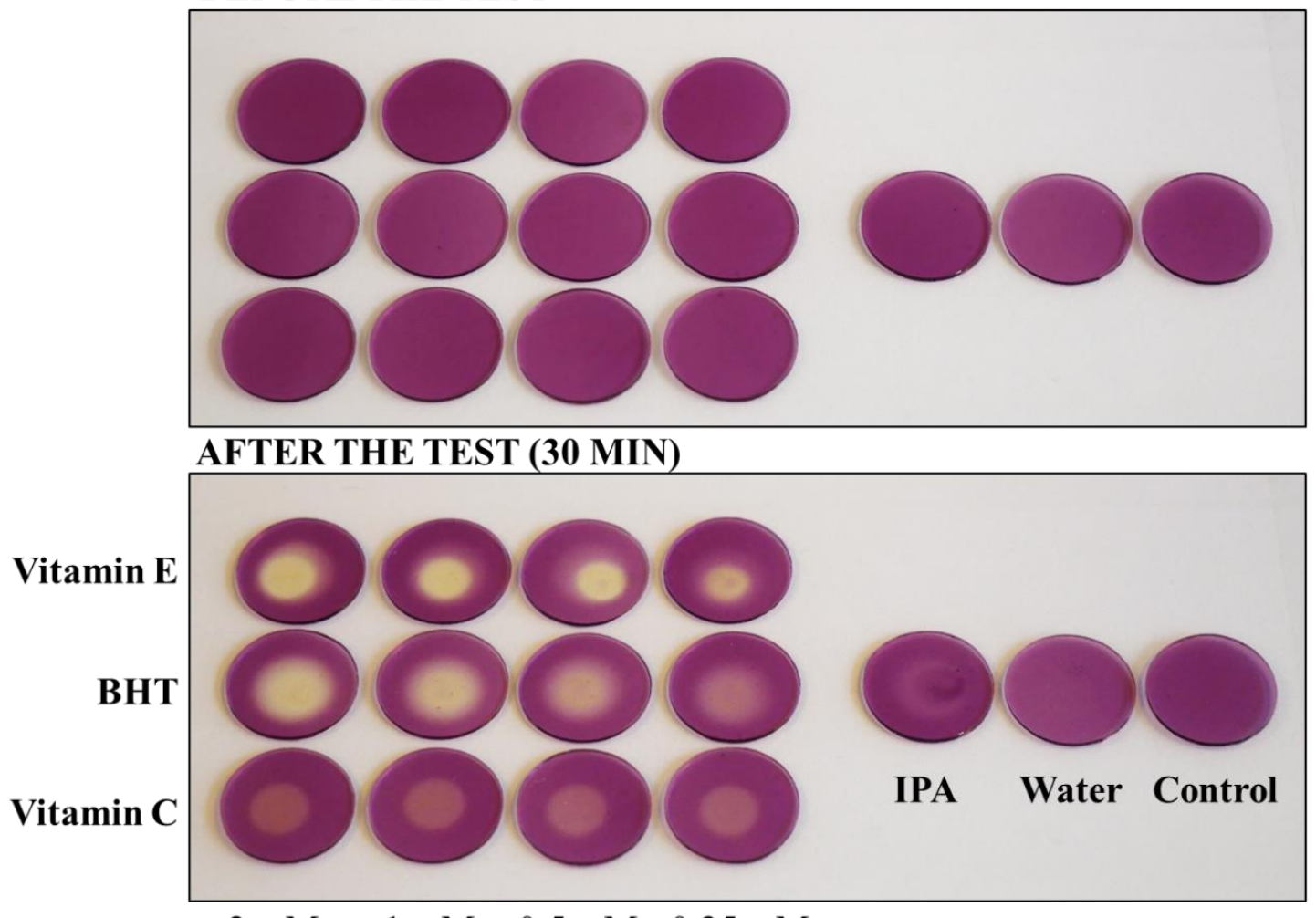

$2 \mathrm{mM} \quad 1 \mathrm{mM} \quad 0.5 \mathrm{mM} \quad 0.25 \mathrm{mM}$

Figure 3. DPPH silicone sensor arrays. The colorimetric sensors are shown before and after dropwise application of $20 \mu \mathrm{L}$ solutions of vitamin C, vitamin E and BHT at four different concentrations. The reference antioxidants scavenge the DPPH radicals entrained in the silicone, and, after the reaction is complete, the discoloration of the sensor can be easily detected and evaluated by the naked eye.

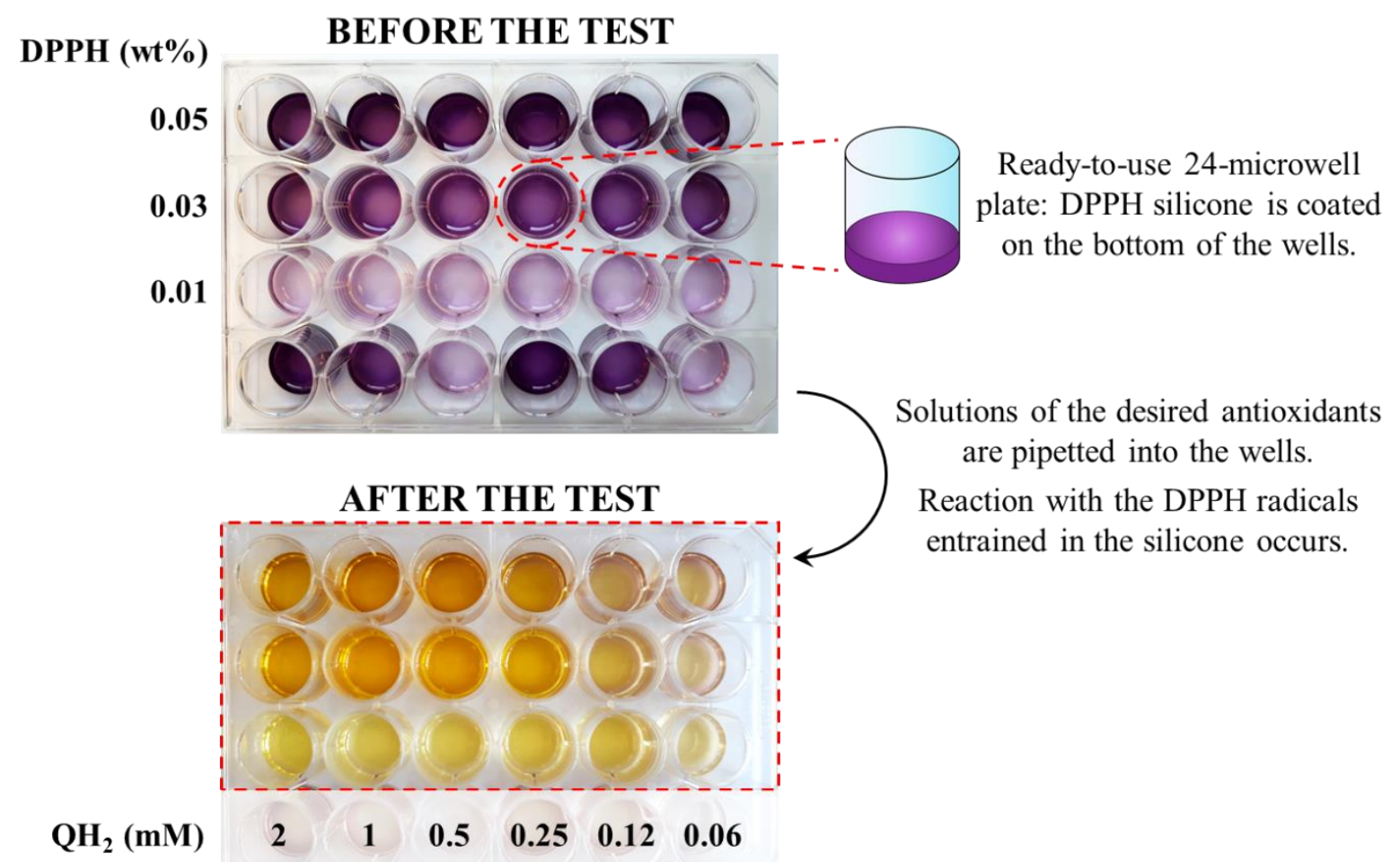


Figure 4. A 24-microwell plate coated with silicone elastomers with immobilized DPPH radicals at three different concentrations $(0.05,0.03$, and $0.01 \mathrm{wt} \%)$. As an example, the plate is shown before and after reaction with quercetin $\left(\mathrm{QH}_{2}\right)$ solutions. $500 \mu \mathrm{L}$ of quercetin solution at six different concentrations $(2,1,0.5,0.25,0.12$, and $0.06 \mathrm{mM})$ are transferred into the wells of the microplate.
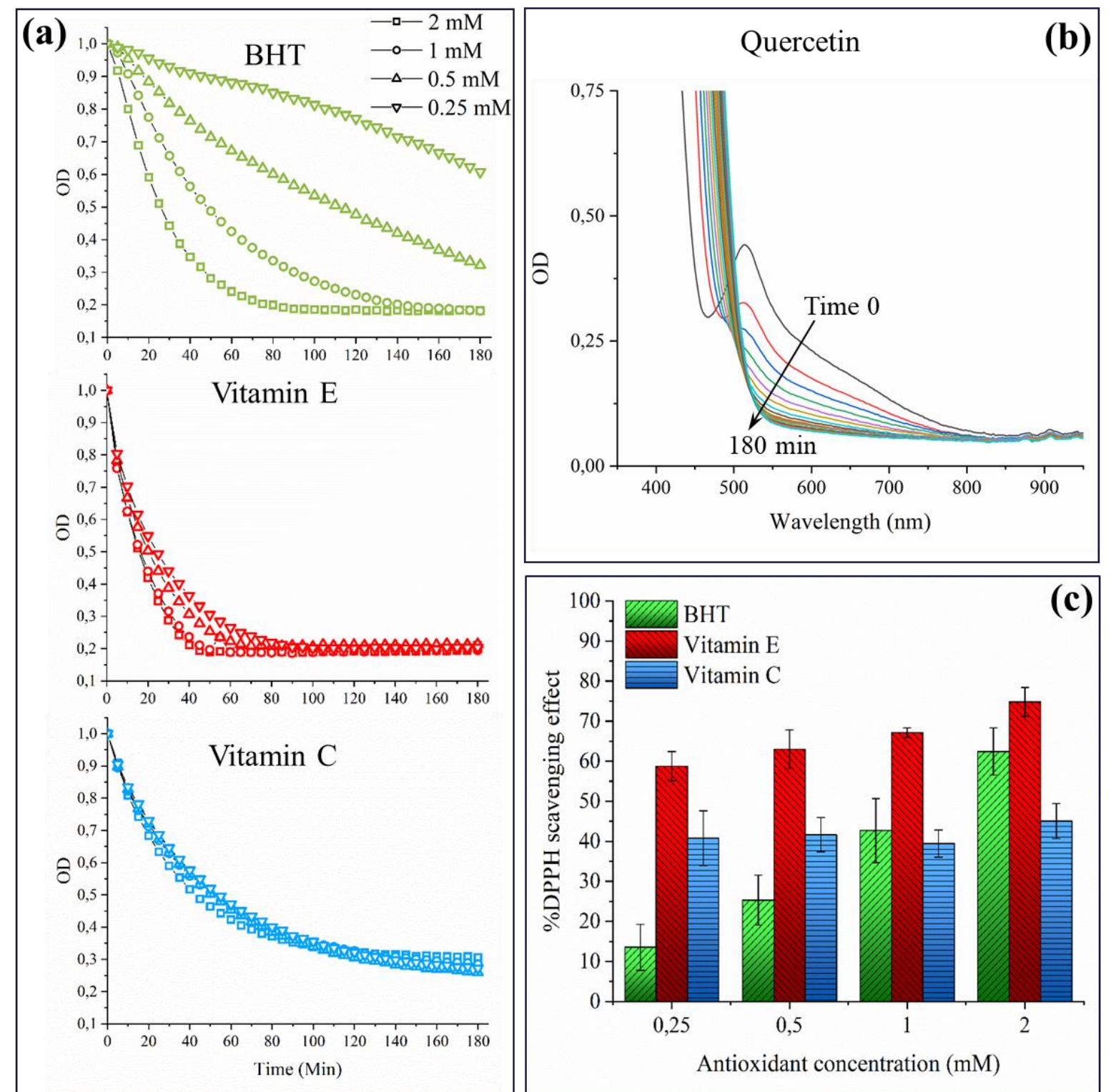

Figure 5. The data reported in the figure correspond to the DPPH silicone sensor with embedded 0.03 wt\% DPPH radicals. a) Response curves of the sensor. Time-dependent decay in absorbance at $514 \mathrm{~nm}$ in response to BHT, vitamin E, and vitamin C solutions at different concentrations. b) Change in the absorbance spectra of the sensor over time in response to a quercetin solution $(2 \mathrm{mM})$. c) \%DPPH scavenging effect corresponding to solutions of BHT, vitamin $\mathrm{E}$, vitamin $\mathrm{C}$, at varying concentrations. 


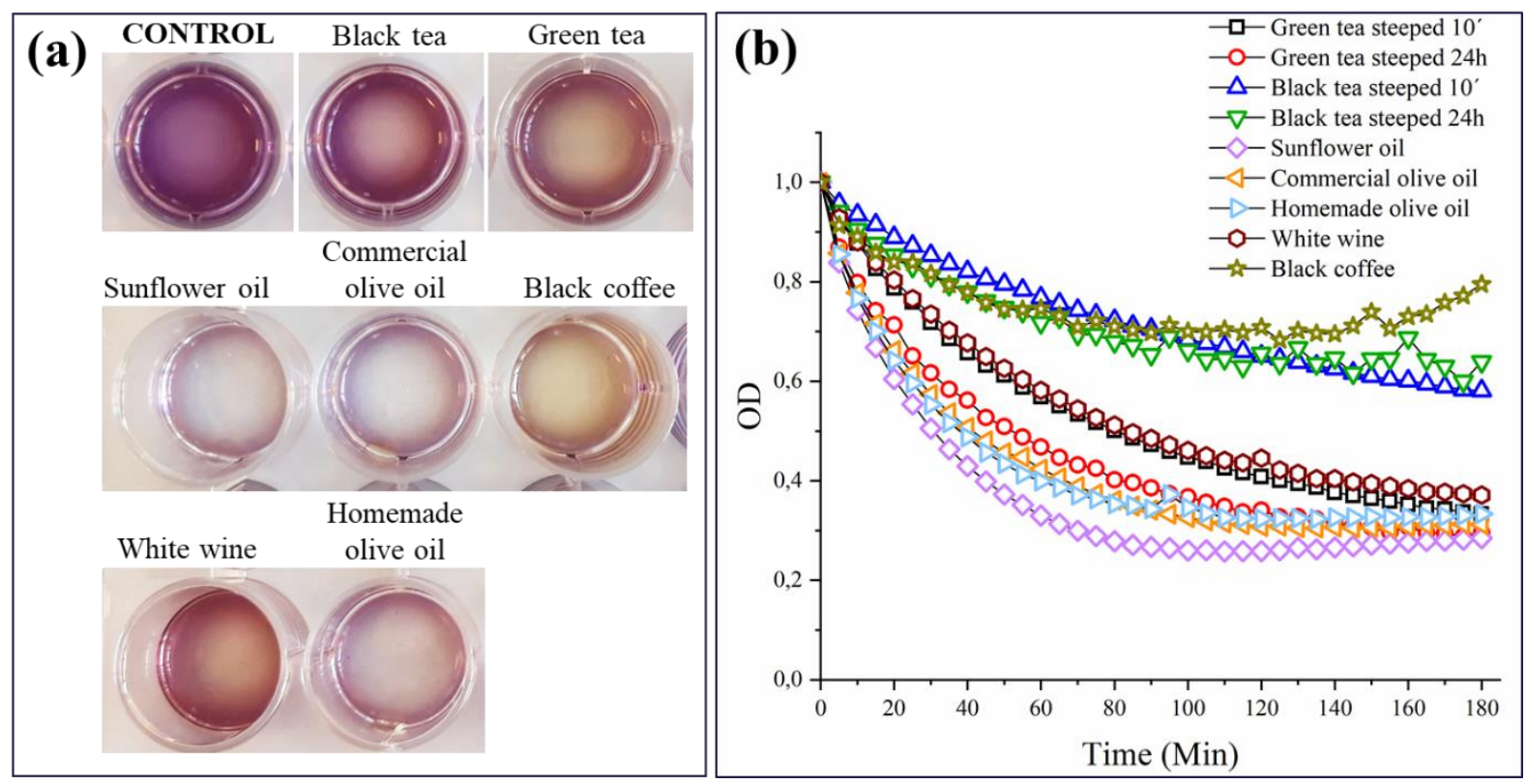

Figure 6. a) DPPH colorimetric sensors coated on the bottom of the wells of a microwell plate are shown after contact with non-pretreated food and beverage samples. b) Response curves of the sensors. Time-dependent decay in absorbance of the DPPH silicone sensor at $514 \mathrm{~nm}$ in response to olive oil, sunflower oil, black tea, green tea, black coffee, and white wine. 
Silicone elastomers with embedded DPPH radicals are used as a portable screening platform for the qualitatively and quantitatively assessment of antioxidant activity of compounds and food samples. The DPPH silicone colorimetric sensor is flexible, highly versatile, and allows for a convenient naked-eye analysis of the results.

Keyword Colorimetric sensor

Elisa Ogliani, Anne Ladegaard Skov*, Michael A. Brook*

Purple to yellow silicone elastomers: design of a versatile sensor for screening antioxidant activity

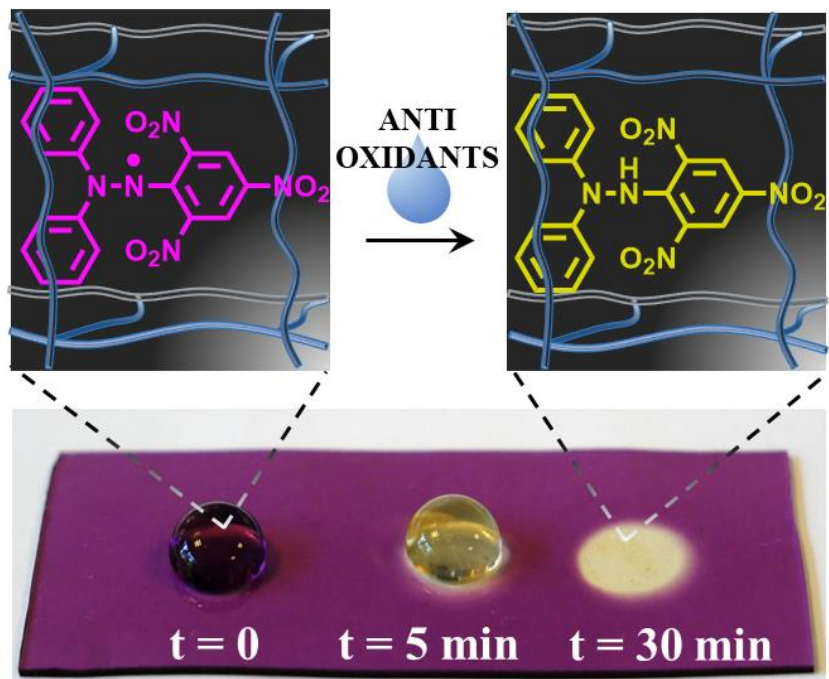

